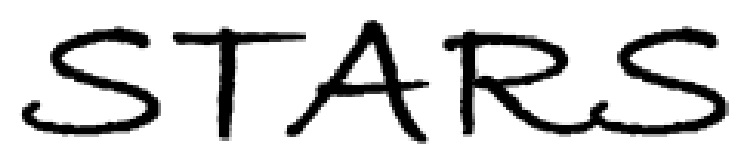

University of Central Florida

STARS

$1-1-2001$

\title{
Nonlinear propagation of two-dimensional gravity wave trains in magnetohydrodynamics
}

David K. Rollins

University of Central Florida

Bhimsen K. Shivamoggi

University of Central Florida

Find similar works at: https://stars.library.ucf.edu/facultybib2000

University of Central Florida Libraries http://library.ucf.edu

This Article is brought to you for free and open access by the Faculty Bibliography at STARS. It has been accepted for inclusion in Faculty Bibliography 2000 s by an authorized administrator of STARS. For more information, please contact STARS@ucf.edu.

\section{Recommended Citation}

Rollins, David K. and Shivamoggi, Bhimsen K., "Nonlinear propagation of two-dimensional gravity wave trains in magnetohydrodynamics" (2001). Faculty Bibliography 2000s. 8182.

https://stars.library.ucf.edu/facultybib2000/8182

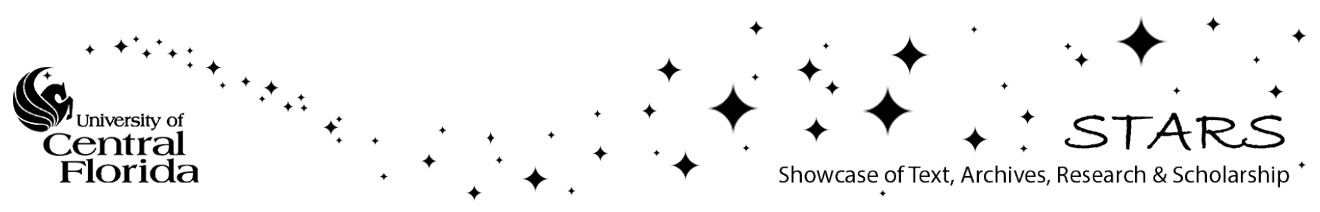




\section{Nonlinear propagation of two- dimensional gravity wave trains in magnetohydrodynamics}

Cite as: Physics of Plasmas 8, 2930 (2001); https://doi.org/10.1063/1.1362528

Submitted: 29 February 2000 . Accepted: 29 January 2001 . Published Online: 23 May 2001

David K. Rollins, and Bhimsen K. Shivamoggi
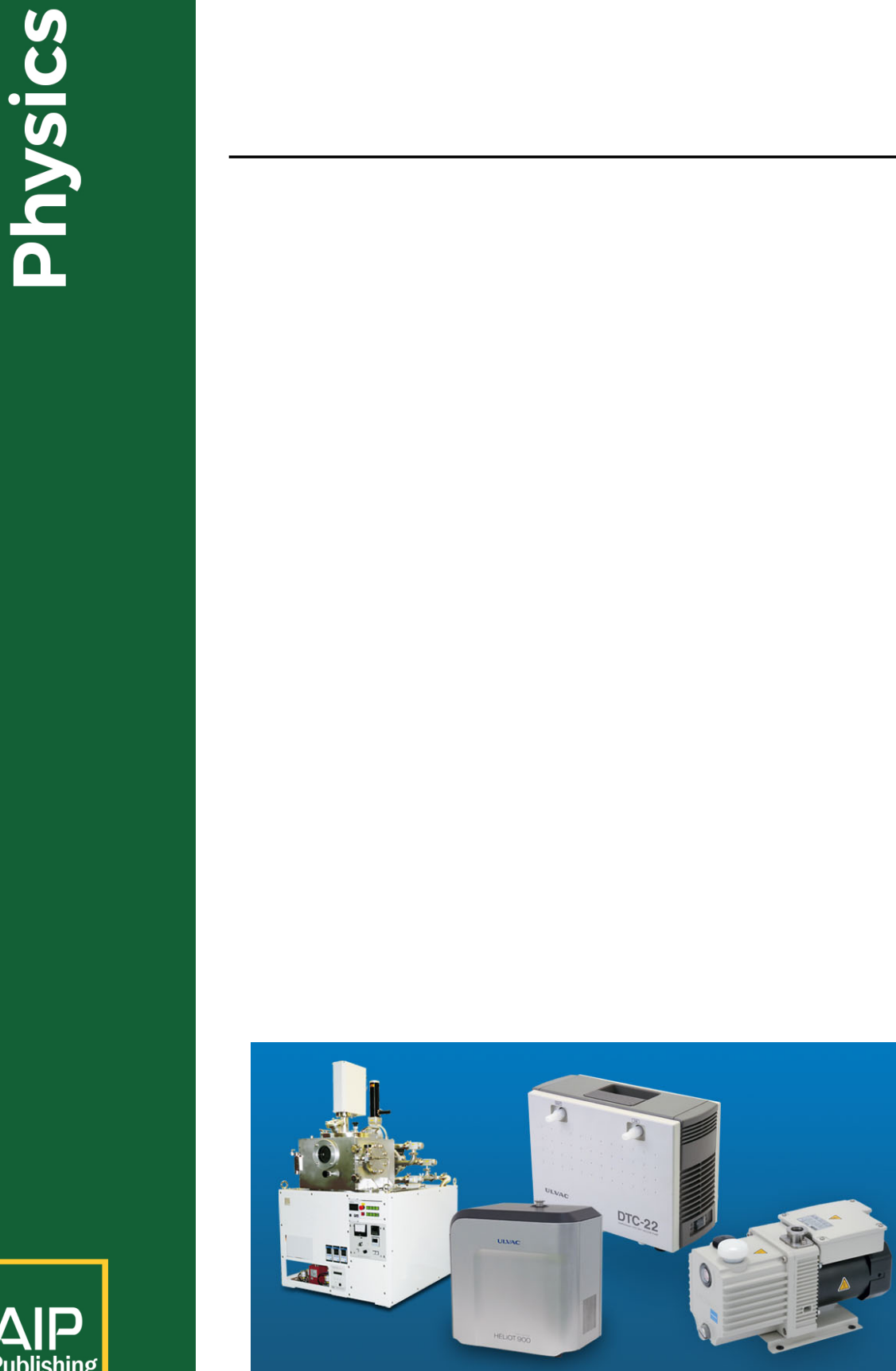

\section{ULVAC}

Leading the World with Vacuum Technology

Vacuum Pumps

- Leak Detectors

Arc Plasma Deposition

RGAs

- Thermal Analysis

Ellipsometers 


\title{
Nonlinear propagation of two-dimensional gravity wave trains in magnetohydrodynamics
}

\author{
David K. Rollins and Bhimsen K. Shivamoggi \\ University of Central Florida, Orlando, Florida 32816
}

(Received 29 February 2000; accepted 29 January 2001)

Nonlinear evolution of modulated two-dimensional gravity wave trains in a conducting fluid subject to a tangential applied magnetic field are considered. The effect of the applied magnetic field on the stability of the modulation and on the saturation amplitude in the long-time evolution are examined.

(C) 2001 American Institute of Physics. [DOI: 10.1063/1.1362528]

\section{INTRODUCTION}

An interface between two plasma media can support low-frequency Alfvén surface waves propagating along the magnetic field in the plane of the interface (Kruskal and Schwarzschild; ${ }^{1}$ Gerwin; ${ }^{2}$ Chen and Hasegawa ${ }^{3,4}$ ). These are similar to gravity wave trains on the surface of water. Alfvén surface waves in a plasma are an important area of research in the context of Alfvén-wave heating of laboratory plasmas (Chen and Hasegawa; $;{ }^{3,4}$ Collins, Cramer, and Donnely; ${ }^{5}$ Appert, Vaclavik, and Villard ${ }^{6}$ ) and solar-atmospheric flux-tube wave propagation, which leads to heating of the solar coronal plasma (Ionson; ${ }^{7}$ Wentzel; ${ }^{8}$ and Roberts ${ }^{9}$ ). The mechanism for the Alfvén-wave heating of laboratory plasmas involves the Alfvén surface waves that are believed (Ref. 4) to provide a means of coupling power from an external antenna to a resonance layer in the plasma. The plasma is then heated by the spatial Alfvén resonance damping of the waves in the plasma.

Studies of one-dimensional surface waves in magnetohydrodynamics in a plane-slab geometry ${ }^{10}$ have been made by Savage, ${ }^{11,12}$ Shivamoggi, ${ }^{13-16}$ Kant and Malik, ${ }^{17}$ among others. In this paper, we consider the nonlinear evolution of the modulated waves in the two-dimensional case, and examine the effect of the applied magnetic field on the nonlinear propagation.

Consider an initially, quiescent, infinitely conducting liquid subjected to a gravitational field in the vertical direction (Fig. 1) that is confined to a region $y=0$ by a vacuum magnetic field aligned with the surface of the liquid. The gravity here may be an effective force field that simulates the dynamic effects due to the curvature of the magnetic field (Rosenbluth and Longmire ${ }^{18}$ ). The dimensionless form of the equations are

$y<\eta: \quad \phi_{x x}+\phi_{y y}+\phi_{z z}=0$,

$y>\eta: \quad \psi_{x x}+\psi_{y y}+\psi_{z z}=0$,

$y=\eta: \quad \phi_{y}=\eta_{t}+\phi_{x} \eta_{x}+\phi_{z} \eta_{z}$,

$\psi_{y}=\eta_{x}+\psi_{x} \eta_{x}+\psi_{z} \eta_{z}$

$\phi_{t}+\frac{1}{2}\left(\phi_{x}^{2}+\phi_{y}^{2}+\phi_{z}^{2}\right)+\eta+M^{2}\left[\psi_{x}+\frac{1}{2}\left(\psi_{x}^{2}+\psi_{y}^{2}+\psi_{z}^{2}\right)\right]=0$ $y \rightarrow \infty: \quad \phi_{y} \rightarrow 0$,

$y \rightarrow \infty: \quad \psi_{y} \rightarrow 0$,

where $\boldsymbol{\nabla} \phi$ is the perturbation in the velocity potential of the liquid, $\nabla \psi$ is the perturbation in the vacuum magnetic field, and $\eta$ is the displacement of the liquid at the interface. Here, we have nondimensionalized the various physical quantities using a reference length $\lambda_{0}$ and a reference time $\sqrt{\lambda_{0} / g}$. The dimensionless parameter $M^{2}=B_{0}^{2} / \lambda_{0} \rho g$, where $B_{0}$ is the magnetic field strength, $g$ is the gravitational acceleration, and $\rho$ is the density of the liquid.

\section{NONLINEAR EVOLUTION EQUATION FOR THE TWO-DIMENSIONAL MODULATED GRAVITY WAVE TRAINS}

The linear dispersion relation $\omega_{0}^{2}=k\left(1+M^{2} k\right)$ can be generalized to the two-dimensional case by interpreting $k$ as the magnitude of the wave vector $k=(l, 0, m)$. This leads to the nonlinear dispersion relation

$$
\omega^{2}=\sqrt{l^{2}+m^{2}}+M^{2}\left(l^{2}+m^{2}\right)+\omega_{0} k_{0}^{2} a^{2} .
$$

Expanding (8) about $k_{0}=\left(k_{0}, 0,0\right)$ with perturbation $\left(k_{1}, 0, k_{2}\right)$ gives

$$
\omega=\omega_{0}+\frac{d \omega_{0}}{d k_{0}} k_{1}-\alpha k_{1}^{2}+\beta k_{2}^{2}+\frac{1}{2} \omega_{0} k_{0}^{2} a^{2},
$$

where

$$
\begin{aligned}
& \omega_{0}=\left(k_{0}+M^{2} k_{0}^{2}\right)^{1 / 2}, \\
& \frac{d \omega_{0}}{d k_{0}}=\left(1+2 M^{2} k_{0}\right) / 2 \omega_{0}, \\
& \alpha=\frac{1}{8} \omega_{0}^{3}, \\
& \beta=\frac{1+2 M^{2} k_{0}}{4 k_{0} \omega_{0}} .
\end{aligned}
$$

Using (9), one may write ${ }^{19}$ the nonlinear Schrödinger equation describing the evolution of the two-dimensional modulated surface waves:

$$
i\left(A_{t}+\frac{d \omega_{0}}{d k_{0}} A_{x}\right)-\alpha A_{x x}+\beta A_{z z}-\kappa\left(|A|^{2}-\left|A_{0}\right|^{2}\right) A=0,
$$




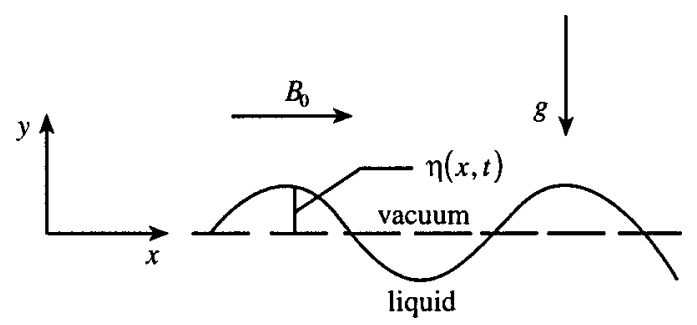

FIG. 1. Deformed surface of plasma supported by a tangential magnetic field.

where

$$
\kappa \equiv \frac{k_{0}^{3}}{2 \omega_{0}}\left(1+2 M^{2} \omega_{0}^{2}\right) .
$$

\section{STABILITY OF TWO-DIMENSIONAL MODULATIONS}

To investigate the stability of the two-dimensional modulations, we put

$$
\begin{gathered}
A(\xi, z, t)=[\rho(\xi, z, t)]^{1 / 2} \exp [i \sigma(\xi, z, t)], \\
\xi=x+\frac{d \omega_{0}}{d k_{0}} t .
\end{gathered}
$$

Equation (11) then leads to

$$
\begin{gathered}
\frac{1}{2} \rho_{t}-\alpha \sigma_{\xi} \rho_{\xi}-\alpha \rho \sigma_{\xi \xi}+\beta \sigma_{z} \rho_{z}+\beta \rho \sigma_{z z}=0, \\
-\rho \sigma_{t}+\frac{\alpha \rho_{\xi}^{2}}{4 \rho}-\frac{\alpha \rho_{\xi \xi}}{2}+\alpha \rho \sigma_{\xi}^{2}-\frac{\beta \rho_{z}^{2}}{\rho}+\frac{\beta \rho_{z z}}{2} \\
-\beta \rho \sigma_{z}^{2}-\kappa \rho\left(\rho-\rho_{0}\right)=0 .
\end{gathered}
$$

Setting further,

$$
\rho=\rho_{0}+\rho_{1}(\xi, z, t), \quad \sigma=\sigma_{1}(\xi, z, t),
$$

linearizing in $\rho_{1}$ and $\sigma_{1}$, and putting

$$
\rho_{1}, \sigma_{1} \sim \exp \left[i\left(K_{1} \xi+K_{2} z-\Omega t\right)\right],
$$

Eqs. (13) and (14) lead to the solvability condition

$$
\Omega^{2}=\left(\alpha K_{1}^{2}-\beta K_{2}^{2}\right)\left(\alpha K_{1}^{2}-\beta K_{2}^{2}-2 \kappa \rho_{0}\right) .
$$

The stability boundaries, given by $\Omega^{2}=0$, are the pair of straight lines,

$$
K_{1}= \pm \sqrt{\frac{\beta}{\alpha}} K_{2}
$$

and the hyperbola

$$
\alpha K_{1}^{2}-\beta K_{2}^{2}-2 \kappa \rho_{0}=0 .
$$

Figure 2 shows the instability region $\left(K_{1}, K_{2}\right)$ space for the applied magnetic field, being zero and nonzero. The linear instability region is unbounded in both cases. However, in

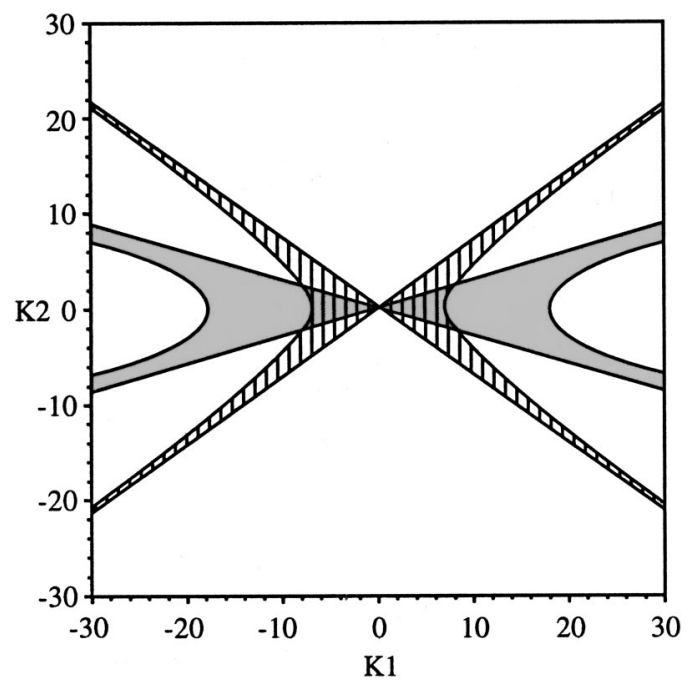

FIG. 2. Unstable region (shaded) in wave number space for zero (bold) and nonzero magnetic parameter $M$.

the MHD case $(M \neq 0)$, the modulations propagating transverse to the applied tangential magnetic field are seen to be stabilized by the latter, as to be expected.

\section{THE LONG-TIME EVOLUTION OF THE MODULATIONALLY UNSTABLE SURFACE WAVES}

We will now consider the effect of the applied tangential magnetic field on the nonlinear development of the initially linearly unstable modulation. For this purpose, we will consider the initial-value problem for modulations with wave numbers near the threshold for instability. The set of hyperbolic branches (19) reduces to the instability threshold $K_{1}$ $= \pm \sqrt{2 \kappa \rho_{0} / \alpha}$ for the one-dimensional modulation. The set of linear branches (18) is peculiar for the two-dimensional modulation and does not exist for the one-dimensional modulation. We need to construct solutions separately near each of the branches.

Following Shivamoggi, ${ }^{20}$ we refine the multiple-scale development given by Janssen ${ }^{21}$ to derive an equation for the nonlinear evolution of the linearly unstable modulation of the gravity wave train. This involves perturbing the wave number $k$ instead of the nonlinearity parameter (as done by Janssen ${ }^{21}$ and inserting in the solutions near the linear instability threshold an explicit detuning parameter $\chi$.

\section{A. Solution near the hyperbolic branches}

We look for a solution of the following form:

$$
\begin{aligned}
& \rho(x, z, \tau)=\rho_{0}+\epsilon \rho_{1}(x, z, \tau)+\epsilon^{2} \rho_{2}(x, z, \tau)+\ldots, \\
& \sigma(x, z, \tau)=\epsilon \sigma_{1}(x, z, \tau)+\epsilon^{2} \sigma_{2}(x, z, \tau)+\ldots, \\
& \alpha K_{1}^{2}-\beta K_{2}^{2}=2 \kappa \rho_{0}+\epsilon^{2} \chi+\ldots,
\end{aligned}
$$

where $\epsilon$ is a small parameter that characterizes the departure of $\alpha K_{1}^{2}-\beta K_{2}^{2}$ from the linear stability threshold value 


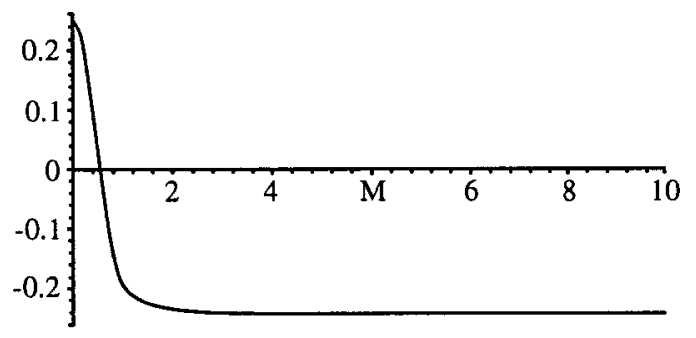

FIG. 3. Saturation amplitude $C$ as a function of the magnetic parameter $M$.

$2 \kappa \rho_{0}, \tau=\epsilon t$ is the show time scale characterizing the slow time evolution near the threshold, and $\chi$ is an explicit detuning parameter.

Substituting (20) into Eqs. (13) and (14), we find that

$$
\rho_{1}(x, z, \tau)=a(\tau) e^{i\left(\sqrt{\alpha} K_{1} x+\sqrt{\beta} K_{2} z\right)}+\text { c.c. },
$$

where c.c. stands for the complex conjugate and $a(\tau)$ is assumed to be real valued. Following the development of Shivamoggi, ${ }^{20}$ we find that $a(\tau)$ satisfies the equation

$$
\left(\frac{d a}{d \tau}\right)^{2}=\kappa^{2}\left(a_{0}^{2}-a^{2}\right)\left(a^{2}-c\right),
$$

where $a_{0}$ is the initial value of $a(\tau)$, and

$$
c \equiv-\frac{2 \alpha \rho_{0} \chi}{\kappa}-a_{0}^{2} .
$$

Equation (22) exhibits periodic, bounded solutions oscillating between 0 and $a_{0}$ if $c<0$, and between $a_{0}$ and $\sqrt{c}$ if $c>0$, which corresponds to $\chi<0$. The latter case implies the saturation of the linearly unstable modulation. Figure 3 shows the variation of the saturation amplitude $c$ with $M^{2}$. Observe that the effect of increasing the magnetic field is to decrease the saturation amplitude $c ; c$ indeed becomes negative if the magnetic field is strong enough.

\section{B. Solution near the linear branches}

We look for a solution of the following form:

$$
\begin{aligned}
& \rho(x, z, \tau)=\rho_{0}+\epsilon \rho_{1}(x, z, \tau)+\epsilon^{2} \rho_{2}(x, z, \tau)+\ldots, \\
& \sigma(x, z, \tau)=\epsilon \sigma_{1}(x, z, \tau)+\epsilon^{2} \sigma_{2}(x, z, \tau)+\ldots, \\
& \alpha K_{1}^{2}-\beta K_{2}^{2}=\epsilon^{2} \chi+\ldots
\end{aligned}
$$

Substituting (23) into Eqs. (13) and (14), we find that

$$
\rho_{1}=0, \sigma_{1}=(\tau) e^{i\left(\sqrt{\alpha} K_{1} x+\sqrt{\beta} K_{2} z\right)}+\text { c.c. }
$$

Following the development of Shivamoggi, ${ }^{20}$ we find that $b(\tau)$ satisfies the equation

$$
\frac{d^{2} b}{d \tau^{2}}-\left(2 \kappa \alpha \rho_{0} \chi\right) b=0 .
$$

Equation (25) shows that nonlinearities to $O\left(\epsilon^{3}\right)$ have no effect on the linearly unstable modulation near the linear branches of the instability threshold.

\section{DISCUSSION}

In this paper, we have considered some aspects of the nonlinear evolution of modulated two-dimensional gravity wave trains in a conducting fluid subjected to a tangential applied magnetic field. We have first investigated the effect of the applied magnetic field on the modulational instability of the surface wave. We have then investigated the effect of the applied magnetic field on the saturation amplitude in the long-time evolution of the modulationally unstable waves. In both investigations, the applied magnetic field is found to have a stabilizing effect in the nonlinear development of the modulated surface waves. This suggests that an applied tangential magnetic field has the potential to play a significant role in the Alfvén-wave heating mechanism of laboratory plasmas which is based on the excitation of Alfvén surface waves by an external coupler and its damping by the spatial Alfvén resonance.

\section{APPENDIX: NONLINEAR EVOLUTION EQUATION FOR THE ONE-DIMENSIONAL MODULATED GRAVITY WAVE TRAINS}

Here we give a derivation of the nonlinear evolution equation describing the modulated surface waves by using the averaged Lagrangian method (Whitham ${ }^{22}$ ). The Lagrangian corresponding to magnetohydrodynamic surface waves is $\left(\right.$ Shivamoggi ${ }^{15}$ )

$$
\begin{aligned}
L= & \int_{-\infty}^{\eta(x, z, t)}\left(\phi_{t}+\frac{1}{2} \phi_{x}^{2}+\frac{1}{2} \phi_{y}^{2}+\frac{1}{2} \phi_{z}^{2}\right) d y \\
& -\int_{\eta(x, z, t)}^{\infty} M^{2}\left(\psi_{x}+\frac{1}{2} \psi_{x}^{2}+\frac{1}{2} \psi_{y}^{2}+\frac{1}{2} \psi_{z}^{2}\right) d y+\frac{1}{2} \eta^{2} .
\end{aligned}
$$

The variational principle,

$$
\delta \int_{t_{1}}^{t_{2}} \int_{x_{1}}^{x_{2}} \int_{z_{1}}^{z_{2}} L d x d z d t=0,
$$

subject to the restrictions that the variations $\delta \phi, \delta \psi$, and $\delta \eta=0$ at the boundaries $x=x_{1}, x_{2}, z=z_{1}, z_{2}$ and $t=t_{1}, t_{2}$ then gives rise to Eqs. (1)-(7) via the usual procedure in the calculus of variations.

We consider a finite-amplitude stationary wave of frequency $\omega_{0}^{2}=k_{0}\left(1+M^{2} k_{0}\right)$ and a wave number $k_{0}$ propagating in the $x$ direction and superpose on it a slowly varying weak modulation. Following Whitham, ${ }^{22}$ we assume that the wave can be taken to be sinusoidal locally, i.e., $\eta=a \cos \theta$, but with amplitude $a$ and $\theta$ phase varying slowly with $x, z$, and $t$, i.e.,

$$
\begin{aligned}
& a=a(x, z, t), \\
& \theta=\theta(x, z, t)=k_{0} x-\omega_{0} t+\sigma(x, z, t) .
\end{aligned}
$$

We may then introduce a generalized frequency $\omega$ and wave numbers $k$ and $l$ via

$$
\begin{aligned}
& \omega=-\theta_{t}=\omega_{0}-\sigma_{t}, \\
& k=\theta_{x}=k_{0}+\sigma_{x}, \quad l=\theta_{z}=\sigma_{z},
\end{aligned}
$$


which satisfy compatibility conditions

$$
k_{t}+\omega_{x}=0, \quad l_{t}+\omega_{z}=0 .
$$

Using Eqs. (1)-(4), (6), and (7), we obtain

$$
\begin{aligned}
\eta= & a \cos \theta+\frac{1}{2} a^{2} k\left(1+2 k M^{2}\right) \cos 2 \theta \\
\phi= & \left(\frac{\omega a}{k} \sin \theta+\frac{\omega a_{x}}{k^{2}}(1-k y) \cos \theta+\frac{a_{t}}{k} \cos \theta\right) e^{k y} \\
& +k \omega a^{2} M^{2} \sin 2 \theta e^{2 k y}, \\
\psi= & \left(a \sin \theta+a_{x} y \cos \theta\right) e^{-k y}+a^{2} \omega^{2} \sin 2 \theta e^{-2 k y} .
\end{aligned}
$$

The long-time evolution equations are found by using (A6)-(A8) in (A1) and calculating the average Lagrangian,

$$
\begin{aligned}
\bar{L}= & \frac{1}{2 \pi} \int_{0}^{2 \pi} L d \theta \\
= & \frac{1}{4}\left(1-\frac{\omega^{2}}{k}\right) a^{2}+\frac{a_{t}^{2}}{4 k}+\frac{a a_{t t}}{2 k}+\frac{\omega a_{t} a_{x}}{4 k^{2}}+\frac{3 \omega^{2} a a_{x x}}{8 k^{3}} \\
& +\frac{3 \omega a a_{x t}}{4 k^{2}}+\frac{\omega^{2} a_{x}^{2}}{8 k^{3}}+\frac{k M^{2} a^{2}}{4}+\frac{1}{8} k^{2} a^{4} \\
& +\frac{1}{4} k^{2} M^{2} \omega^{2} a^{4}-\frac{M^{2} a_{x}^{2}}{8}-\frac{M^{2} a a_{x x}}{8 k} .
\end{aligned}
$$

Variation of $\bar{L}$ with respect to $\theta$ gives

$$
\frac{\partial}{\partial t}\left(a^{2}\right)+\frac{\partial}{\partial x}\left(\frac{d \omega_{0}}{d k_{0}} a^{2}\right)=0,
$$

and variation of $\bar{L}$ with respect to $a$ gives

$$
\omega=\omega_{0}\left[1+\frac{a_{x x}}{8 \omega_{0}^{4} a}+\frac{k^{3}}{2 \omega_{0}^{2}}\left(1+2 M^{2} \omega_{0}^{2}\right) a^{2}\right] .
$$

For weak modulations, expanding (A11) about $k=k_{0}$ and $a$ $=0$, we have

$\omega=\omega_{0}+\frac{d \omega_{0}}{d k_{0}}\left(k-k_{0}\right)+\frac{1}{2} \frac{d^{2} \omega_{0}}{d k_{0}^{2}}\left[\left(k-k_{0}\right)^{2}-\frac{a_{x x}}{a}\right]+\frac{\partial \omega}{\partial a^{2}} a^{2}$.

Using (A5), we obtain from (A10) and (A12), the coupled equations,

$a_{t}+\frac{d \omega_{0}}{d k_{0}} a_{x}+\frac{1}{2} \frac{d^{2} \omega_{0}}{d k_{0}^{2}}\left(\sigma_{x x} a+2 \sigma_{x} a_{x}\right)=0$,

$$
\begin{gathered}
\sigma_{t}+\frac{d \omega_{0}}{d k_{0}} \sigma_{x}+\frac{1}{2} \frac{d^{2} \omega_{0}}{d k_{0}^{2}}\left(\sigma_{x}^{2}-\frac{a_{x x}}{a}\right) \\
+\frac{k_{0}^{3}}{2 \omega_{0}}\left(1+2 M^{2} \omega_{0}^{2}\right) a^{2}=0 .
\end{gathered}
$$

Putting $A=a e^{i \sigma}$ in (A13) and combining (A13) and (A14), we obtain the nonlinear Schrödinger equation

$$
\begin{gathered}
i\left(A_{t}+\frac{d \omega_{0}}{d k_{0}} A_{x}\right)+\frac{1}{2} \frac{d^{2} \omega_{0}}{d k_{0}^{2}} A_{x x}-\frac{k_{0}^{3}}{2 \omega_{0}} \\
\times\left(1+2 M^{2} \omega_{0}^{2}\right)|A|^{2} A=0 .
\end{gathered}
$$

The derivation of Eq. (A15) was given by Kant and Malik ${ }^{17}$ using, on the other hand, the method of multiple scales $\left(\right.$ Kevorkian and $\mathrm{Cole}^{23}$ ).

${ }^{1}$ M. D. Kruskal and M. Schwarzschild, Proc. R. Soc. London, Ser. A 223, 348 (1954).

${ }^{2}$ R. Gerwin, Phys. Fluids 10, 2164 (1967).

${ }^{3}$ L. Chen and A. Hasegawa, J. Geophys. Res. 79, 1033 (1974).

${ }^{4}$ L. Chen and A. Hasegawa, Phys. Fluids 17, 1399 (1974).

${ }^{5}$ G. A. Collins, N. F. Cramer, and I. J. Donnely, Plasma Phys. Controlled Fusion 26, 273 (1984).

${ }^{6}$ K. Appert, J. Vaclavik, and L. Villard, Phys. Fluids 27, 432 (1984).

${ }^{7}$ J. A. Ionson, Astrophys. J. 226, 650 (1979).

${ }^{8}$ D. G. Wentzel, Astron. Astrophys. 76, 20 (1979).

${ }^{9}$ B. Roberts, Sol. Phys. 69, 27 (1981).

${ }^{10}$ The plane-slab geometry is a reasonable approximation for tokamak configuration, if one identifies the $x$ axis with the local magnetic field direction, the $y$ axis with the radial coordinate, and the $z$ axis with the azimuthal coordinate (Fig. 1).

${ }^{11}$ M. D. Savage, J. Plasma Phys. 1, 229 (1967).

${ }^{12}$ M. D. Savage, J. Fluid Mech. 42, 289 (1970).

${ }^{13}$ B. K. Shivamoggi, J. Plasma Phys. 27, 321 (1982).

${ }^{14}$ B. K. Shivamoggi, J. Plasma Phys. 28, 13 (1982).

${ }^{15}$ B. K. Shivamoggi, Q. Appl. Math. 41, 31 (1983).

${ }^{16}$ B. K. Shivamoggi, J. Plasma Phys. 43, 183 (1990).

${ }^{17}$ R. Kant and S. K. Malik, Phys. Fluids 28, 3534 (1985).

${ }^{18}$ M. N. Rosenbluth and C. L. Longmire, Ann. Phys. (N.Y.) 1, 120 (1957).

${ }^{19}$ This procedure is substantiated by a detailed calculation in the Appendix where the one-dimensional version of Eq. (11) is derived by using the averaged Lagrangian method [Whitham (Ref. 20)]. The extension to the two-dimensional case is straightforward, but the algebra is involved.

${ }^{20}$ G. B. Whitham, Proc. R. Soc. London, Ser. A 299, 6 (1967).

${ }^{21}$ B. K. Shivamoggi, J. Phys. A 23, 4289 (1990).

${ }^{22}$ P. A. E. M. Janssen, Phys. Fluids 24, 23 (1981).

${ }^{23}$ J. Kevorkian and J. D. Cole, Perturbation Methods in Applied Mathematics (Springer-Verlag, Berlin, 1981). 